INNOVACIÓN

\title{
Aportes para la enseñanza universitaria de la historia reciente: Una revisita a las memorias de la dictadura argentina a través de dispositivos audiovisuales
}

\author{
Contributions to the teaching of recent history at universities: \\ Revisiting the memories of Argentina's dictatorship through audiovisual devices
}

\author{
Nicolás Fernando Gómez \\ Universidad Nacional de General Sarmiento, Argentina
}

\begin{abstract}
RESUMEN Enseñar lo acontecido en la última dictadura militar en Argentina representa constantes desafíos en las escuelas, pero enseñar a quienes pronto serán docentes involucra un orden distinto de responsabilidades. Atendiendo a estos problemas, en este artículo nos proponemos abordar una secuencia didáctica destinada a ser implementada en las universidades o instituciones de formación docente que tengan carreras afines al campo de la historia. Para ello, incorporamos el uso de recursos audiovisuales que resultan potentes en la complejización de la mirada sobre hechos de «carácter traumático» de la historia reciente en Argentina. En este marco, el artículo propone identificar las distintas memorias sobre la dictadura que circulan en las décadas de 1980, 1990 y 2000, en particular las que son hegemónicas en cada período. Estos elementos nos permiten evidenciar que las memorias nos «dicen» más sobre la coyuntura en las que son recuperadas, y por ende construidas, que sobre aquello que se recuerda. Finalmente, este análisis dará cuenta también de las relaciones entre política estatal y memorias oficiales. Acompañar a nuestros estudiantes en la comprensión de estos procesos de rememoración de un pasado cercano traumático invita a deconstruir lo que damos por sentado y lo que naturalizamos cuando miramos el presente.
\end{abstract}

PALABRAS CLAVE Secuencia didáctica, universidad, recursos audiovisuales, memorias de la dictadura, historia reciente.

ABSTRACT Teaching what happened during the last military dictatorship in Argentina represents a number of challenges at secondary schools, but teaching those who are soon to become teachers demands a different degree of responsibility. Taking this issue into account, this paper attempts to work on a didactic unit designed to be carried out at universities or teaching training institutions that offer courses or studies related to the 
field of history. With that purpose in mind, we incorporate the use of audiovisual devices as powerful tools to deconstruct our reading of traumatic events of recent history in Argentina. Within this framework, the paper identifies the different memories from the time of the dictatorship that coexist during the 1980s, 1990s and 200os, especially those that are hegemonic in each period. We attempt to show that these memories tell us more about the context in which they were produced and constructed rather than about what was actually remembered. This analysis will also account for the relationship between public policy and official memories. Understanding these processes of recollection of a traumatic recent past helps us deconstruct those issues that we take for granted and those we naturalize when we look at the present.

KEYWORDS Didactic unit, university, audiovisual devices, memories of the dictatorship, recent history.

\section{Introducción}

A un año del cuadragésimo aniversario del golpe militar en Argentina, resulta particularmente relevante revisar no sólo las distintas representaciones del gobierno de facto (Arnoso-Martínez, Arnoso-Martínez y Pérez Sales, 2012), sino también las políticas de memorias llevadas adelante por los gobiernos democráticos posteriores al proceso. La enseñanza de este particular proceso histórico y de las implicancias todavía actuales en la sociedad argentina configura el núcleo duro de este artículo.

El proceso dictatorial abierto en la Argentina en 1976 es pasible de ser comprendido a partir de una lectura que atienda al contexto internacional y local: específicamente, las coyunturas de los demás países del Cono Sur, los intereses de Estados Unidos y la escalada de violencia política de nuestro país en los momentos previos al golpe. Las dificultades del gobierno depuesto (presidencia de María Estela Martínez de Perón $)^{1}$ para otorgar sentido al Estado, la persecución paraestatal inaugurada con la Triple A (Alianza Anticomunista Argentina) ${ }^{2}$ junto con el descalabro de la economía y la sed de revancha social por parte de grandes grupos económicos orquestaron el contexto mediato al golpe. Esta polifonía de factores y agentes fueron revisados con mayor o menor énfasis en los distintos ensayos que buscaron dar cuenta de los orígenes y las causas de la última dictadura militar, que mostraron las particularidades

1. María Estela Martínez de Perón asumió la presidencia constitucional de Argentina en 1974 tras la muerte de su esposo y presidente Juan Domingo Perón, durante el ciclo histórico que ha dado en llamarse «tercer peronismo».

2. La Alianza Anticomunista Argentina, conocida como «Triple A», fue un grupo parapolicial organizado y financiado desde el Ministerio de Bienestar Social, a cargo de López Rega, junto con distintos grupos de derecha que participaron en la represión llevada a cabo a partir de 1974. 
frente al ciclo de «intervenciones» de las fuerzas armadas ${ }^{3}$ como las continuidades en torno a la lógica de la violencia y del «enemigo político». Es así como la construcción de legitimidad de lo excepcional se convirtió en una de las tareas primordiales de las fuerzas armadas frente a la sociedad de entonces. Pensar las representaciones sobre el proceso que confluyeron hasta nuestros días nos permite comprender la permanencia de determinados discursos sobre el pasado reciente que operan sobre el sentido común de amplios y heterogéneos sectores de la sociedad.

\section{Una posible estrategia de abordaje en la universidad}

En el ámbito de la enseñanza universitaria no existen recetas que nos garanticen un recorrido totalmente efectivo en la transmisión de saberes. Cuando el proceso histórico que tenemos que enseñar presenta heridas todavía abiertas, el desafío se redobla. Atendiendo a estos problemas, en el presente artículo abordaremos una secuencia didáctica pensada para llevarse a cabo en las universidades o instituciones de formación docente ${ }^{4}$ que tengan carreras afines al campo de la historia. Si bien puede desplegarse en una variedad de espacios académicos, es potencialmente deseable implementarla en las cátedras vinculadas a la historia contemporánea o reciente argentina cuyos programas de estudios incorporen el proceso dictatorial de 1976. Un segundo eje que sostiene esta propuesta es la incorporación de recursos audiovisuales que habilitan un abordaje distinto al de los materiales bibliográficos tradicionales. Estos fragmentos audiovisuales están disponibles libremente en la web y el docente universitario puede proyectarlos durante el desarrollo de la clase con el objetivo de complejizar el análisis sobre el período trabajado.

En este sentido, se requerirá de los estudiantes la lectura previa de determinados documentos al momento de la proyección de los videos, de modo que cuenten con una disponibilidad de herramientas teóricas para el abordaje de dichos fragmentos. El material de lectura previa para los estudiantes que asistan a la clase les permitirá bordear los principales marcos teóricos que habilitan el abordaje de las memorias de la dictadura, para luego ponerlas en función de nuevos interrogantes y dispositivos que se ofrezcan en el seminario. Dentro de la propuesta didáctica presente, el libro $\mathrm{La}$

3. En total se realizaron seis golpes de Estado en la Argentina durante el siglo XX, en 1930, 1943, 1955, 1962,1966 y 1976.

4. Argentina ofrece a los estudiantes que decidan cursar el profesorado en Historia la posibilidad de elegir entre dos opciones dentro de la estructura educativa de la formación superior: por un lado, pueden optar por instituciones superiores de formación docente (anteriormente institutos terciarios) o por universidades nacionales que ofrezcan esta carrera. Ambas opciones presentan un amplio abanico de instituciones de gestión privada y pública y son las únicas habilitadas para la formación de profesores que se insertaran en el sistema educativo argentino. 
cambiante memoria de la dictadura (2008) de Daniel Lvovich y Jaquelina Bisquert ${ }^{5}$ funciona de columna vertebral, ya que el eje propuesto para abordar los fragmentos audiovisuales se enmarcan en las memorias de los ochenta, los noventa y los 2000. Por otro lado, los recursos audiovisuales corresponden a: 1) fragmentos de la película La historia oficial (1985), dirigida por Luis Puenzo; 2) el video documental Los vecinos del horror (1996), llevado a cabo por un grupo de investigadores que recogieron testimonios de vecinos que convivían junto a centros clandestinos de detención durante la dictadura; y 3) el primer capítulo del documental Espacios de memoria, titulado «Vecinos del horror», cuya producción data del 2011 y fue realizado para el canal Encuentro. ${ }^{6}$

Estos videos no tienen como finalidad explicitar las políticas de memoria estatales de los gobiernos sucesivos a la dictadura, ya que los dos primeros objetos de análisis no corresponden a producciones llevadas adelante por el propio Estado Nacional, razón por la cual entorpecería cualquier intento de comparación que se intente desplazar por ese eje. El objetivo es que los alumnos puedan abordar las distintas memorias que conviven o coexisten en los relatos que aparecen en la totalidad de los videos, y ensayar especulaciones en torno a las intenciones de los enunciadores que toman la palabra. La propuesta tiene como hipótesis que estos discursos resultan, por lo menos, sintomáticos de la coyuntura política que reconstruye el texto de Lvovich y Bisquert. Es así que se espera la identificación por parte de los alumnos de las tensiones y disputas en las distintas memorias que perforan la arena pública, las contradicciones al interior de cada discurso, así como también los cambios y continuidades en los argumentos.

\section{Reflexiones en torno a la necesidad de abordar las representaciones sociales}

Los discursos que aparecen tanto en la bibliografía como en los materiales audiovisuales permiten pensar simultáneamente las «actitudes sociales» de por lo menos una parte del arco social frente a la última dictadura militar. Los posicionamientos y la capacidad de agencia de la sociedad coetánea a la dictadura se encuentran íntimamente relacionados con las distintas representaciones del gobierno militar que ella sostenía. Esa mirada que la sociedad de fines de los setenta y principios de los ochenta tenía de

5. Este libro forma parte de la colección « 25 años, 25 libros», editada por la Universidad Nacional de General Sarmiento, y es el material de lectura obligatorio que deben traer los alumnos para la clase en cuestión. Se puede descargar gratuitamente desde el sitio web de la universidad, disponible en http://bit. ly/2kioN6b.

6. Sebastián Schindel, «Espacios de la memoria - Virrey Ceballos: Vecinos del horror», 2014, disponible en https://youtu.be/KıU-GFUxmx8. Encuentro es un canal televisivo de Argentina que emite desde 2007 y, hasta el año 2016, dependía del Ministerio de Educación, Ciencia y Tecnología. El mismo se caracteriza por la transmisión de conocimiento de variados campos educativos, científicos y culturales. 
su propio presente estaba lejos de ser estática, ya que acudió a transformaciones supeditadas a las distintas etapas de «acercamiento-distanciación» a la dictadura militar. El gobierno de facto, por su parte, buscó legitimar su accionar, entre otras cosas, a partir del contraste con el «caos» de los últimos años democráticos y de colocarse en el lugar de «salvador de la nación». De acuerdo con la lectura de Lvovich, una transformación significativa en las representaciones del pasado dictatorial se dio «tras la derrota de Malvinas [cuando] el conjunto del régimen militar fue puesto en cuestión, y el pasado inmediato resultó objeto de una marcada reinterpretación» (Lvovich y Bisquert, 2008: 25; véase también Carassai, 2017; Da Silva Catela, 2015). Esto nos permite desandar una lectura monolítica que recrea una sociedad argentina abroquelada en una sola actitud, o acorralada por el terror que no daba lugar a disidencias. El fracaso bélico, sumado a las dificultades económicas y la creciente denuncia de los organismos internacionales «comenzaban a borrar de las memorias los diversos grados y modos de apoyo que distintos sectores civiles habían dado el régimen militar» (Lvovich y Bisquert, 2008: 25).

Las distintas representaciones que se fueron dando en las últimas décadas en torno a la relación sociedad-golpe militar constituyen intentos por explicar lo sucedido y pugnan por adquirir un estatuto de «verdad». Estas representaciones contienen contradicciones que se transforman con el tiempo y que dependen de políticas de memoria estatales, de los discursos dominantes, entre otros factores. Como explica Elizabeth Jelin (2002), la memoria selecciona y recorta las formas del recuerdo y el olvido en función de instancias sociales obteniendo una clara función performativa del pasado reciente. Por su parte, la centralidad del concepto de memoria reivindicado por los organismos de derechos humanos, por ejemplo, constituye una forma de representación del pasado capaz de definir identidades. Abordar estas tensiones nos obliga a revisar la relación entre historia y memoria, ya que en su diferenciación en torno a la representación del pasado es donde encontramos potentes claves de lectura.

En línea con lo anterior, en otro artículo de Lvovich (2009) se amplía el panorama al indicar las tensiones en torno a los apoyos con los que contó el proceso militar y las distintas interpretaciones que la historiografía fue tejiendo al respecto. Las dificultades para emplear la noción de «consenso» no tardan en aparecer, y el planteo se complejiza cuando se interpela a vecinos que lindaban los centros clandestinos de detención. Al respecto, la falta de manifestaciones de oposición o resistencia no puede ser sencillamente equiparada a un consenso tácito motivado por la aprobación de las políticas del régimen (Lvovich, 2009). Este elemento, por ejemplo, jugará un papel importante en el análisis del fragmento de Los vecinos del horror (Levín, 2005), ya que allí se encuentran algunos relatos polémicos que intentan recrear las implicancias de convivir con el terror. Con esto, cabe mencionar que los relatos de memoria de los ochenta, los noventa y los 2000 poco nos pueden decir sobre la experiencia de «vivir» 
en los setenta; su principal potencial radica en aquellas representaciones que nacen después de mucho tiempo y que aluden a aquello que «vivieron» durante la dictadura.

\section{Las memorias de la dictadura como objeto de la enseñanza universitaria}

La secuencia didáctica que propongo en este artículo es producto de distintos ensayos y experiencias en el marco del Seminario en Investigación en Historia, espacio curricular del profesorado en Historia de la Universidad Nacional de General Sarmiento 7 . Durante estos años en el Espacio de Trabajo sobre Historia Reciente ${ }^{8}$ hemos reflexionado en torno a las prácticas docentes universitarias que resultan significativas para la enseñanza de procesos históricos de carácter traumático y reciente. Esta propuesta, por lo tanto, no pretende mostrarse como acabada, sino más bien como un posible camino para recorrer en la enseñanza de procesos históricos recientes, atendiendo a las características propias de las universidades como también a los actores que intervienen en ella, especialmente a los destinatarios de nuestros esfuerzos pedagógicos: los propios estudiantes universitarios.

En la fase de apertura de la clase sugerimos iniciar el recorrido con un capítulo del libro Los trabajos de la memoria de Elizabeth Jelin que los estudiantes pueden abordar en grupos finalizada la presentación e introducción del docente. En este sentido, se puede introducir el problema a partir de preguntas que habiliten a los estudiantes el ensayo de posibles respuestas apoyándose en sus propios recorridos eruditos; un primer movimiento puede consistir en arrojar el interrogante «¿Qué es la memoria?», cuya ganancia puede resultar en la propia manifestación de las dificultades que tienen algunas definiciones o caracterizaciones en torno a la «memoria». Algunas preguntas que pueden apuntalar las ideas que traen los estudiantes pueden ser: ¿cómo funciona la memoria? ¿Podemos confiar/creer en ella? ¿Las memorias siempre recuerdan hechos «reales»? ¿Es posible trasmitir un recuerdo? ¿Por qué la memoria recuerda algunas cosas y otras no? ¿Qué relación existe entre la memoria y el olvido? ¿El olvido es necesariamente perjudicial? ¿En qué consiste la separación entre «memoria individual» $\mathrm{y}$ «memoria colectiva»? ¿Qué podemos reflexionar en torno a la «transmisión»? A continuación, proponemos que el docente realice la presentación del material

7. La Universidad Nacional de General Sarmiento (UNGS) está emplazada en la ciudad de Los Polvorines, Provincia de Buenos Aires. Dicha casa de estudios tiene veintinco años desde su creación y posee un claro perfil que la vincula fuertemente con el territorio que la rodea, tanto en la formación de sus estudiantes como en los proyectos que lleva adelante. Se encuentra cerca al ex Centro Clandestino de Detención de Campo de Mayo.

8. El Espacio de Trabajo sobre Historia Reciente (ETHR) está conformado por investigadores, docentes, adscriptos y becarios de la Universidad Nacional de General Sarmiento bajo la dirección de la doctora Florencia Levín. Este equipo de trabajo lleva adelante distintas actividades de discusión y formación académica vinculadas al campo de la historia reciente argentina. 
«¿De qué hablamos cuando hablamos de memorias?» (Lvovich y Jelin, 2008) de Elizabeth Jelin o bien cualquier otro texto que el docente a cargo considere pertinente para la conceptualización de la memoria. Al finalizar esta secuencia, el docente puede optar por repartir en grupos el artículo de Jelin con el objetivo de promover las discusiones en torno a las nociones que el texto ofrece. Para ello, se debe brindar un espacio de trabajo en grupo y ofrecer una serie de consignas orientativas, de modo que los estudiantes puedan ajustar su abordaje a partir de distintas claves de lectura. Algunas de ellas pueden ser: ¿Cuáles son las características de la memoria individual y colectiva? ¿Por qué la autora sostiene que «lo social» siempre está presente en la memoria, inclusive en aquella que parece más íntima? ¿Qué cuestiones aparecen en la construcción de memorias? ¿Cómo aparece la «identidad» en estas discusiones? ¿Cómo se define la noción de «trauma»? ¿Cómo se vincula con los procesos de memoria? ¿Que implican los «períodos calmos»y los «períodos de crisis» para las memorias sociales? Si tuvieras que traerlo a la actualidad, ¿Qué etapa estaríamos atravesando? ¿Por qué? ¿Qué rol cumple el «olvido» dentro de los marcos constructivos de memoria?

Este tipo de procedimientos permiten que el docente visibilice el horizonte de su clase y explicite a sus estudiantes las lecturas a las que se espera logren arribar. Asimismo, también habilita la apropiación de algunas discusiones que resultan potentes para el análisis de los videos. Durante este tiempo de discusión el rol del docente consiste en recorrer las distintas islas de trabajo para acompañar la lectura de los estudiantes. También puede resultar beneficioso que el docente tome apuntes de las principales dificultades que observa, las cuestiones que se repiten y dudas comunes, etcétera. Una vez concluido el trabajo grupal, se cierra este primer bloque con la puesta en común, tomando como eje las preguntas orientativas y las observaciones que el docente fue registrando del trabajo en equipo.

\section{Los recursos audiovisuales en la enseñanza de las memorias. Una propuesta didáctica}

En el segundo bloque de la clase proponemos trabajar con los recursos audiovisuales, lo que permite a los estudiantes poner en función lo discutido anteriormente. Las potencialidades de trabajar con estos dispositivos radican en que permiten una mayor «creatividad» $\mathrm{y}$ «libertad» en la interpretación de los sentidos de la obra. Si bien en el nivel universitario el uso de estos recursos parecería más «extraño», la utilización de los mismos resulta beneficioso en la comprensión de ciertos aspectos de los fenómenos «excepcionales». La cercanía de este pasado y la filmación de los mismos contribuyen a la recreación del contexto histórico que merecen ser aprovechados, ya que no siempre contamos con la posibilidad de ver a los sujetos de la historia frente a una cámara, tomando la palabra y construyendo sentidos para sus contemporáneos. Es así que estos productos habilitan la multiperspectiva ante sus espectadores, 
lo que permite «recrear» otras dimensiones o registros históricos y educar la mirada de nuestros estudiantes (Dussel, 2006: 277-293; véase también Cela-Ranilla y otros, 2017; Rodríguez Torres, 2014; Pérez de la Cruz y Pérez de la Cruz, 2016). Este «revivir» de los actores cobra nuevos sentidos en cada interpelación que lo revisita y recorre; en particular, nuestros estudiantes podrán mirar estos fragmentos con la coyuntura de un nuevo aniversario de la dictadura.

Para iniciar, se dará un recorrido por La cambiante memoria de la dictadura presentando su estructura y desarrollando una exposición dialogada que incorpore los aportes de los estudiantes. Vale aclarar que, si bien en la clase se aborda el libro en su totalidad, en la presente secuencia se recorre la obra de manera gradual y cronológica respetando la secuencia «décadas de ochenta, noventa y 2000». Los fragmentos audiovisuales que corresponden a cada década son los que trazan la estructura de la exposición. La intención es que los estudiantes puedan colaborar en la reconstrucción del texto, de manera que este modelo explicativo está sujeto a cambios y, lógicamente, no implica que el docente sea el único que comente la obra.

\section{Aproximaciones a las representaciones de la memoria en los ochenta, noventa y 2000. Un abordaje desde los recursos audiovisuales en el nivel superior}

\section{Década del ochenta: La historia oficial}

El capítulo que principalmente nos va a ofrecer herramientas para la década de 1980 es el titulado «La transición democrática y la teoría de los dos demonios (1983-1986)» (páginas 27 a 43). Recomendamos que el docente comience explicando los principales procesos y el contexto de esta década. $\mathrm{Al}$ respecto cabe mencionar que, durante los ochenta, las representaciones de la dictadura impulsadas por el primer gobierno postdictadura de Ricardo Alfonsín giraron en torno a lo que se conoce como «teoría de los dos demonios», inaugurada oficialmente en el prólogo del Nunca más. ${ }^{9}$ Allí, los desaparecidos eran presentados como víctimas inocentes, despojados de su politicidad como también de la condición de «subversivos» que la dictadura se había encargado de pregonar. En esta escena simbólica, la sociedad operaba con «inocencia» en «la explicación de lo sucedido en los años setenta como responsabilidad y resultado de dos violencias» totalmente ajenas a ella (Franco, 2012: 29; véase también Muñoz, 2017). Cabe mencionar que esta etapa se encuentra marcada por las tensiones del gobierno de Alfonsín para lograr satisfacer las demandas sociales y mantener una mínima «cordialidad» con las fuerzas militares. Es así que, en primer término, se

9. Nombre adoptado en 1984 por la Comisión Nacional sobre la Desaparición de Personas (CONADEP) para titular el informe final sobre la investigación de los crímenes cometidos por la dictadura militar. El mismo fue publicado como libro en sucesivas ediciones. 
aprobaron una serie de medidas para responder a las demandas de una sociedad que ahora se aferraba a los ideales democráticos y la defensa de los derechos humanos. No obstante, en los medios de comunicación se utilizaron estas banderas sociales para emprender lo que se denominó el «show del horror», que implicaba un morboso aprovechamiento mediático de las pruebas que iban apareciendo día a día.

Al respecto, Lvovich señala que la «teoría de los dos demonios» abonó a la estabilidad democrática en tanto sistema de pluralidad de opiniones y resolución de conflictos en un marco de legalidad, idea que perdura hasta nuestros días en distintas manifestaciones y discursos. Esta memoria no tardó en volverse hegemónica y rápidamente fue naturalizada y replicada por los medios de comunicación, perforando espacios artísticos como el teatro y el cine. En ese contexto se origina la película que proponemos analizar en este artículo: La historia oficial (1985), que pone de manifiesto la existencia de una sociedad inocente e ignorante de lo que pasaba a su alrededor. En los fragmentos elegidos, se puede apreciar claramente que ni siquiera la esposa de un empresario vinculado a los militares «sabía» lo que sucedía, aun cuando se trataba de una persona «formada» en su calidad de profesora de Historia. Los autores mencionan que, en este contexto, los largometrajes La noche de los lápices (1986) y La historia oficial son ejemplos de la memoria de la represión tal como se concebía en ese entonces.

Finalizada la explicación y el análisis del capítulo que aborda los ochenta, recomendamos proyectar los fragmentos seleccionados de la película La historia oficial solicitando a los estudiantes que tengan presente lo discutido y leído. Es potencialmente productivo recomendar que tomen nota de aquellas escenas que les remitan a algún proceso o fenómeno abordado por la bibliografía para que obtengan más precisiones en el momento de la puesta en común. Asimismo, es muy potente que el docente establezca un espacio de debate para que los estudiantes puedan evidenciar en la película las conceptualizaciones sobre la memoria, y las características de los ochenta trabajados en la clase. Finalmente, una consigna disparadora que puede implementarse es solicitar a la audiencia que preste especial atención a los actores sociales que aparecen representados en el film, que intenten recrear sus imaginarios, sus pertenencias sociales, sus contradicciones y los argumentos que sostienen sus prácticas.

\section{Década del noventa: Los vecinos del horror}

Finalizada la discusión anterior se abordará la década del noventa, con la misma estructura anterior: primero un abordaje del texto de lectura sugerido en este artículo y después la proyección del fragmento audiovisual correspondiente a dicho periodo. El capítulo del libro de Lvovich y Bisquert que recorre esta década se titula «Un pasado que no pasa (1987-1995)» (páginas 45 a 57) y vislumbra los años noventa bajo la ges- 
tión del presidente Carlos Saúl Menem. Pone en el eje de la cuestión la inauguración de una política de "pacificación nacional» impulsando la Ley de Obediencia Debida ${ }^{10}$ e indultos ${ }^{11}$ con el objetivo explícito de "cerrar las heridas del pasado». Allí se gesta la «teoría de la reconciliación nacional» que colocaba al terrorismo estatal en el mismo plano que el «subversivo». En esta operación, ambos eran igualados, aunque de manera distinta que en los ochenta, a partir de la interpelación a los «dos demonios» para que reconocieran «aciertos y errores».

La memoria que se impulsa entonces pone el énfasis en dejar el pasado para «avanzar hacia el futuro», con una lógica discursiva que sostenía la imagen clínica de la «herida suturada» asociada a las ideas del olvido y el entierro de los conflictos con el «único fin», declaraba el gobierno, de eliminar las «divisiones». Los autores muestran a partir de estos hechos qué tan conflictiva resultaba ser la memoria sobre la dictadura que, pese a la creciente hegemonía de la teoría de los dos demonios frente a amplios sectores de la sociedad, todavía encontraba duras resistencias que reivindicaban lo actuado en «la guerra sucia». En este proceso, los organismos de derechos humanos perdieron capacidad de convocatoria y prácticamente salieron de la agenda presidencial. Una decisión en sintonía con estos discursos fue el resarcimiento económico a las víctimas del terrorismo de Estado, como un intento por mostrar «imparcialidad» frente a los «dos demonios».

Para abordar este período sugerimos la proyección del video realizado por investigadores de psicología de la Universidad de Buenos Aires en el año 1996, titulado Los vecinos del horror. ${ }^{12}$ Se trata de un material por demás valioso, ya que está realizado con testimonios de vecinos «comunes» que vivían lindantes a ex centros clandestinos de detención (Calveiro, 1998; véase también Colombo, 2011). Resulta interesante entonces explorar las distintas representaciones e imaginarios que aparecen de la experiencia dictatorial veinte años más tarde. Para explotar el recurso, sugerimos que, al momento de mirar cada testimonio, los estudiantes tomen nota de los discursos que emiten, pero también de sus actitudes, muecas y silencios que dejan escapar. Por otro lado, es importante que identifiquen si algunas de las memorias trabajadas aparecen explicita o tácitamente en los relatos, como también las contradicciones que puedan darse en un mismo testimonio.

10. La Ley de Obediencia Debida establecía la inocencia jurídica de ciertos rangos y grados militares por actuar bajo las órdenes de escalafones superiores.

11. Los indultos fueron una serie de diez decretos sancionados entre el 7 de octubre de 1989 y el 30 de diciembre de 1990 por el entonces presidente de la Argentina Carlos Menem, los cuales indultaban a civiles y militares que cometieron crímenes durante la dictadura incluyendo a los miembros de las juntas condenados en el Juicio a las Juntas de 1985, al procesado ministro de Economía José Alfredo Martínez de Hoz y los líderes de las organizaciones guerrilleras.

12. Este material tiene ya veinte años y fue realizado en formato VHS. Es por ello que la calidad de imagen y sonido no son los óptimos. 
Las posibles preguntas que pueden orientan la lectura del video son las siguientes: ¿qué impresión tienen de cómo era la convivencia entre el campo y la vecindad? ¿Qué contradicciones aparecen en los testimonios? A partir de estos testimonios, ¿qué podemos saber nosotros acerca de «lo que sabían o no sabían» estos vecinos que vivieron cerca de los centros clandestinos de detención? ¿Qué relación se puede establecer, a propósito de estos testimonios, entre conocimiento y consenso? ¿Y entre conocimiento y terror? ¿Encuentran algún vínculo entre los testimonios de los vecinos y algunas nociones estudiadas en esta clase?

\section{Los 2000: Espacios de memoria}

Por su parte, el inicio del nuevo siglo representa un complejo período de avances y retrocesos en materia judicial, lo que resultó en una diferenciación entre cada extremo de la década del 2000. La relevancia social del terrorismo de Estado se puso de manifiesto en cada 24 de marzo a partir de actos multitudinarios, aunque no sin mostrar la persistencia del discurso militar tradicional en algunos sectores de la sociedad argentina. En este último período se corporizan las tensiones y polémicas en torno a los espacios de memoria que venían diagramándose en la recta final del menemismo. En este sentido, cobraron vigor las iniciativas para cimentar lugares de la memoria, como los llama Jelin, junto con la conformación de archivos, la construcción de monumentos y el señalamiento simbólico de los lugares vinculados a la represión ilegal (Lvovich y Bisquert, 2008). Estos lugares de memoria son espacios donde se intenta materializar la memoria. En el capítulo «Las políticas de memoria del Estado (2003-2007)» de Lvovich y Bisquert se realiza un recorrido panorámico de la primera gestión del Kirchnerismo en materia de memoria. Para finalizar, los autores recorren el año 2006 destacando los múltiples actos a propósito del trigésimo aniversario del golpe militar. Allí se establece el 24 de marzo como feriado nacional y, ese mismo año, el presidente Néstor Kirchner autoriza el pleno acceso a los archivos militares de la dictadura. Es interesante remarcar también que en este marco comienza a circular la noción de cívico-militar a la hora de denominar el proceso abierto en 1976, junto con la reedición del Nunca más con nuevo prólogo alineado al pensamiento oficial (Crenzel, 2015; véase también Lorenz, 2002).

A este respecto es que se incorpora el último material a trabajar en la clase: el documental del canal Encuentro que corresponde al primer capítulo del ciclo Espacios de memoria, titulado "Virrey Ceballos: Vecinos del horror», cuya producción data del 2011. Allí los estudiantes podrán analizar los discursos de los «vecinos del horror» durante el 2011, lo que permitirá comparar estas representaciones con las del video de 1996. A diferencia de los otros fragmentos, aquí se les cede la palabra a sobrevivientes de Centros Clandestinos de Detención (CCD) y es el Estado Nacional el que lo lleva adelante para luego ser emitido en un canal público. Finalizada la proyección, se 
recomienda iniciar el último espacio de debate y puesta en común de esta secuencia didáctica. Como este periodo en particular llega hasta nuestros días, recomendamos que los docentes habiliten las reflexiones sobre las políticas de memorias actuales, las disputas en torno a la visibilidad de algunas memorias, como así también el rol de los medios de comunicación en la aparición de determinas representaciones.

\section{Cierre y balance final}

Este recorrido panorámico de las distintas memorias habilita a que las reflexiones del cierre queden en manos de los propios estudiantes a partir de una serie de preguntas abiertas. El objetivo final es que los estudiantes puedan comprender cabalmente aquello que ya traían leído y que mencionen las perspectivas o sensaciones que produjeron la elección de estos tres fragmentos audiovisuales. Esta actividad permite articular distintos procesos de aprendizaje y reforzarlos en la medida que los estudiantes vayan sumando sus propias perspectivas. Las distintas experiencias de ensayo de esta secuencia nos indican que generalmente los estudiantes aportan anécdotas o situaciones de su propia vida cotidiana o familiar, lo cual se traduce en el ejercicio de vincular estas experiencias con los procesos de memoria colectiva que sugerimos transitar durante esta secuencia. Las preguntas disparadoras para este momento final pueden ser: ¿toda memoria oficial es necesariamente hegemónica? ¿Hasta qué punto podemos «creer» en los distintos testimonios? ¿Qué tipo de conocimiento podríamos construir con estos testimonios? ¿Qué memorias permanecieron durante la temprana postdictadura hasta la actualidad? ¿Cuál podría ser la memoria hegemónica en la actualidad? ¿Qué implicancias tuvo conservar la teoría de los dos demonios? ¿Y en la actualidad, qué sucede con esta memoria/teoría?

Finalmente, es importante destacar que, en tanto estudiantes de un profesorado, estos contenidos resultan relevantes para su formación teórica y pedagógica, ya debido a la incorporación de reflexiones vigentes en nuestro contexto inmediato, como por la utilización de recursos audiovisuales que permiten educar la mirada de nuestros estudiantes. En particular, consideramos que nuestros estudiantes, en tanto futuros docentes de Historia, tienen que atender a estas memorias en las aulas, lo que demanda una formación teórica y discursiva especialmente sensible sobre estos temas. Por otro lado, no podemos dejar de insistir en la importancia de incorporar recursos audiovisuales que contribuyen a complejizar el análisis y que, en tanto formato distinto al que estamos acostumbrados docentes y estudiantes universitarios, habilitan otros registros vinculados al análisis estético y artístico. 


\section{Referencias}

Arnoso-Martínez, Maitane, Ainara Arnoso-Martínez y Pau Pérez-Sales (2012). «Representaciones sociales del pasado: La dictadura militar argentina en la memoria colectiva». Revista de Psicología Social, 27 (3): 259-272. DOI: 10.1174/021347412802845540.

Calveiro, Pilar (1998). Poder y desaparición: Los campos de concentración en la Argentina. Buenos Aires: Colihue.

Carassai, Sebastián (2017). «Violencia política, dictadura militar y memoria. La Argentina de los años setenta y ochenta a partir del Archivo Marshall T. Meyer». Estudos Ibero-Americanos, 43 (2): 380-396. DOI: 10.15448/1980-864X.2017.2.25164.

Cela-Ranilla, José María, Vanessa Esteve González, Francesc Esteve Mon, Juan GonzÁlez Martínez y Mercè Gisbert-Cervera (2017). «El docente en la sociedad digital: Una propuesta basada en la pedagogía transformativa y en la tecnología avanzada». Profesorado, 21 (1): 403-422. Disponible en http://bit.ly/2iq4JKW.

Crenzel, Emilio (2015). «Genesis, uses, and significations of the Nunca Más report in Argentina». Latin American Perspectives 42, $\mathrm{n}^{\circ} .3$ (2015): 20-38. DOI:

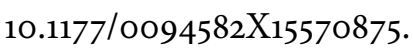

Cоцомво, Pamela (2011). «Espacio y desaparición: Los campos de concentración en Argentina». Isegoría, 45: 639-652. DOI: 10.3989/isegoria.2011.i45.747.

Da Silva Catela, Ludmila (2015). «Staged memories: Conflicts and tensions in Argentine public memory sites». Memory Studies, 8 (1): 9-21. DOI: $10.1177 / 1750698014552403$.

Dussel, Inés (2006). «Educar la mirada. Reflexiones sobre una experiencia de producción audiovisual y de formación docente». En Inés Dussel y Daniela Gutiérrez (editoras), Educar la mirada. Políticas y pedagogías de la imagen. Buenos Aires: Manantial.

Franco, Marina (2012). Un enemigo para la nación: Orden interno, violencia $y$ «subversión», 1973-1976. Buenos Aires: Fondo de Cultura Económica.

JeLIN, Elizabeth (2002). Los trabajos de la memoria. Madrid: Siglo XXI.

Levín, Florencia (2005). «Arqueología de la memoria. Algunas reflexiones a propósito de Los vecinos del horror. Los otros testigos». Revista Entrepasados, 15 (28).

Lorenz, Federico (2002). «¿De quién es el 24 de marzo? Las lucha por la memoria del golpe de 1976». En Elizabeth Jelin (compiladora), Las conmemoraciones en las fechas in-felices. Buenos Aires: Siglo XXI.

Lvovich, Daniel (2009). «Sistema político y actitudes sociales en la legitimación de la dictadura militar argentina (1976-1983)». Ayer, 75: 275-299. Disponible en http:// bit.ly/2BNhhEU. 
LVOVICH, Daniel y Jaquelina BISQuert (2008). La cambiante memoria de la dictadura militar desde 1984: Discursos públicos, movimientos sociales y legitimidad democrática. Buenos Aires: UNGS/Biblioteca Nacional.

MuÑoz, María Antonia (2017). «La(s) violencia(s) y la democracia como conceptos co-dependientes: El caso argentino». Athenea Digital, 17 (2): 3-30. DOI: 10.5565/ rev/athenea.1554.

Pérez de la CruZ, Francisco Javier y Sagrario Pérez de LA CruZ (2016). «Implementación docente de materiales audiovisuales en diferentes ramas del conocimiento». Opción, 32 (12): 212-237. Disponible en http://bit.ly/2BKzy5z.

RODríguez TORRES, Javier (2014). «Diseño de materiales y tecnología. Itinerarios hacia la innovación». Historia y Comunicación Social, 19: 675-687. DOI: 10.5209/ rev_HICS.2014.v19.45169.

\section{Reconocimiento}

Esta secuencia didáctica es el producto final del Seminario de Enseñanza de la Historia, en el marco de la Maestría en Historia Contemporánea dictada por la Universidad Nacional de General Sarmiento, Argentina.

\section{Sobre el autor}

Nicolás Fernando Gómez es profesor universitario de Educación Superior en Historia por la Universidad Nacional de General Sarmiento, actualmente cursante de la Maestría en Historia Contemporánea por la misma universidad. Becario en docencia e investigación en el Seminario de Investigación en Historia (IDH-UNGS). Su correo electrónico es ngomez@ungs.edu.ar. 


\title{
REVISTA PEDAGOGÍA UNIVERSITARIA Y DIDÁCTICA DEL DERECHO
}

La Revista Pedagogía Universitaria y Didáctica del Derecho (RPUDD) es una publicación científica semestral que contribuye tanto a la reflexión multidisciplinaria sobre Pedagogía Universitaria y Didáctica del Derecho, para la formación y consolidación de esta área de investigación, como a la difusión de prácticas innovadoras en la enseñanza-aprendizaje del Derecho, considerando el contexto nacional e internacional..

\author{
DIRECTORA \\ María Francisca Elgueta Rosas \\ ASISTENTE EDITORIAL \\ Priscilla Saavedra Medina \\ SITIO WEB \\ pedagogiaderecho.uchile.cl \\ E-MAIL \\ rpedagogia@derecho.uchile.cl \\ LICENCIA DE ESTE ARTÍ́CULO
}

Creative Commons Atribución Compartir Igual 4.0 Internacional

\author{
9 \\ La edición de textos, el diseño editorial \\ y la conversión a formatos electrónicos de este artículo \\ han estado a cargo de Tipográfica \\ (www.tipografica.cl).
}

